\title{
A Simple Baseline for Pose Tracking in Videos of Crowded Scenes
}

\author{
Li Yuan* \\ Shuning Chang* \\ Ziyuan Huang* \\ yuanli@u.nus.edu \\ National University of Singapore \\ Xuecheng Nie \\ YITU Technology
}

\author{
Yichen Zhou \\ YITU Technology \\ National University of Singapore \\ Francis E.H. Tay \\ National University of Singapore \\ Shuicheng Yan \\ YITU Technology
}

\author{
Yunpeng Chen \\ YITU Technology
}

\begin{abstract}
This paper presents our solution to ACM MM challenge: Large-scale Human-centric Video Analysis in Complex Events [13]; specifically, here we focus on Track3: Crowd Pose Tracking in Complex Events. Remarkable progress has been made in multi-pose training in recent years. However, how to track the human pose in crowded and complex environments has not been well addressed. We formulate the problem as several subproblems to be solved. First, we use a multi-object tracking method to assign human ID to each bounding box generated by the detection model. After that, a pose is generated to each bounding box with ID. At last, optical flow is used to take advantage of the temporal information in the videos and generate the final pose tracking result.
\end{abstract}

\section{KEYWORDS}

pose tracking, human detection, human tracking, pose estimation, human in events

ACM Reference Format:

Li Yuan, Shuning Chang, Ziyuan Huang, Yichen Zhou, Yunpeng Chen, Xuecheng Nie, Francis E.H. Tay, Jiashi Feng, and Shuicheng Yan. 2020. A Simple Baseline for Pose Tracking in Videos of Crowded Scenes. In Proceedings of the 28th ACM International Conference on Multimedia (MM '20), October 12-16, 2020, Seattle, WA, USA. ACM, New York, NY, USA, 5 pages. https://doi.org/10.1145/3394171.3416300

\section{INTRODUCTION}

The general pipeline for the pose tracking method that we used can be divided into two parts, respectively human tracking and

*Authors contributed equally to this work; Work done during internship at YITU Technology.

Permission to make digital or hard copies of all or part of this work for personal or classroom use is granted without fee provided that copies are not made or distributed for profit or commercial advantage and that copies bear this notice and the full citation on the first page. Copyrights for components of this work owned by others than ACM must be honored. Abstracting with credit is permitted. To copy otherwise, or republish, to post on servers or to redistribute to lists, requires prior specific permission and/or a fee. Request permissions from permissions@acm.org.

MM '20, October 12-16, 2020, Seattle, WA, USA

(C) 2020 Association for Computing Machinery.

ACM ISBN 978-1-4503-7988-5/20/10 . \$15.00

https://doi.org/10.1145/3394171.3416300 pose estimation. First, we use a multi-object tracking method to assign an id to each human boxes generated by the detection phase. During human tracking, some detection boxes will be refined or deleted. In the second step, we perform pose estimation on every box that is assigned an id during tracking. The result of the pose estimation combined with the id assigned by the tracking method is the final submission result. The overall structure of our method can be seen in Fig. 1.

Since the problem is treated as a two-stage problem to be tackled one by one, each module will be introduced separately. The following of the report is organized as follows: Sec. 2 introduces the human detection model that we used; Sec. 3 introduces the human tracking algorithm; Sec. 4 introduces the pose estimation as well as the final pose generation process. Finally, Sec. 5 concludes the report.

\section{HUMAN DETECTION}

The first step of human pose tracking is to detect the bounding boxes of person. As no validation set in HIE dataset [13], we split the original training set as a new training set and validation set. Two splitting strategies are tried: splitting by image frames (5k for validation, $27 \mathrm{k}$ for training) and splitting by videos. We found that splitting by image will cause over-fitting and far away from the data distribution of the testing set. So we adopt the video-splitting strategy and split video 3,7,8 and 17 as the validation set and the rest videos as train data, in which $5.7 \mathrm{k}$ image frames for validation and the reset $27 \mathrm{k}$ images frames for training. Based on the train and validation set, we can conduct detection experiments on HIE. All the performance of models is tested by two metrics, Averaged Precision (AP) and MMR [3]. AP reflects both the precision and recall ratios of the detection results; MMR is the log-average Miss Rate on False Positive Per Image (FPPI) in [0.01,100], is commonly used in pedestrian detection. MR is very sensitive to false positives (FPs), especially FPs with high confidences will significantly harm the MMR ratio. Larger AP and smaller MMR indicates better performance. 


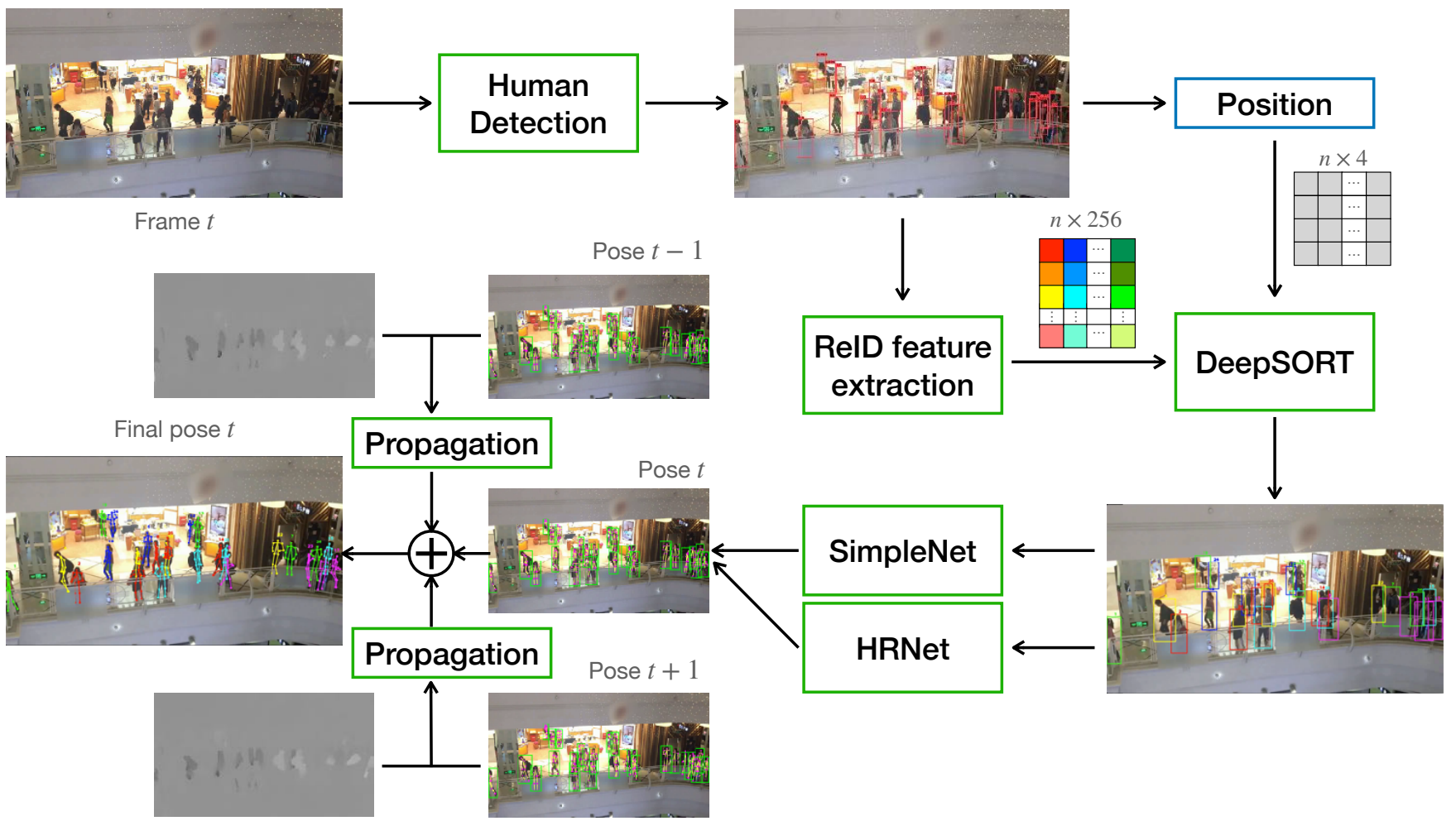

Figure 1: The overall structure of the proposed solution to human pose tracking. The solution is formulated as two stages. In the first stage, humans in every video frames are detected and DeepSORT is applied to associate boxes in different frames. For DeepSORT tracking, we used our own pretrained ReID model. Combined with position information, DeepSORT is able to perform well on the test set of HIE. Second stage involves human pose estimation as well as tracking. The pose estimation is performed on each box in the tracking result. Taking advantage of the pose in the last frame as well as the subsequent frame, the pose of the current frame is smoothed temporally. The identity of the box is automatically transferred to the final poses with the corresponding bounding boxes.

\subsection{Detection Smoothing by Optical Flow}

For human tracking in video, smoothing bounding boxes on human instances can be beneficial to reduce the tracking miss rate. We propose to smooth the current bounding box of human instance based on the previous frame by optical flow. Give one detected bounding box with joints coordinates set $I_{i}^{k}$ (including two points: $\left(x_{1}, y_{1}\right)$ for upper right point and $\left(x_{2}, y_{2}\right)$ as lower left point) in current frame $J^{k}$, we compute the $I_{i}^{k-1}$ in frame $J^{k-1}$, and the optical flow field $F^{k-1 \rightarrow k}$ between the two consequent frames. Then the prediction joints coordinates set $\hat{I}_{i}^{k}$ can be estimated by the optical flow field $F^{k-1 \rightarrow k}$ and the detection coordinate $I_{i}^{k-1}$ in last frame, i.e. $\hat{I}_{i}^{k}=I_{i}^{k-1}+F^{k-1 \rightarrow k}$. Similar with the Kalman filter, the current position is the weight sum of prediction and observations, thus we have the "real" bounding box in current frame is weight sum of detected box and estimated box: $\tilde{I}_{i}^{k}=\alpha \hat{I}_{i}^{k}+(1-\alpha) I_{i}^{k}$, where $\alpha$ is a weight and it is 0.5 in most of cases.

\subsection{Common Methods on HIE}

There are mainly two different types of common detection frameworks: one-stage (unified) frameworks [14-16] and two-stage (regionbased) framework [4-6, 17]. Since RCNN [5] has been proposed, the two-stage detection methods have been widely adopted or modified $[1,9,11,17,21,22,28]$. Normally, the one-stage frameworks can run in real-time but with the cost of a drop in accuracy compared with two-stage frameworks, so we mainly adopt two-stage frameworks on HIE dataset.

We first investigate the performance of different detection backbone and framework on HIE dataset, including backbone: ResNet152 [7], ResNeXt101 [25] and SeNet154 [8], and different framework: FasterRCNN [17], Cascade R-CNN [1], and Feature-Pyramid Networks (FPN) [10]. The experimental results on different backbone and methods are given in Table 1. The baseline model is Faster RCNN with ResNet50, and we search hyper-parameters on the baseline model then apply to the larger backbone. From table 1, we can find that the better backbone (ResNet152 and ResNeXt101) and combining advanced methods (Cascade and FPN) can improve the detection performance, but the SENet154 does not get better performance than ResNet152 even it has superior classification performance 
on ImageNet. So in our final detection solution, we only adopt ResNet152 and ResNeXt101 as the backbone.

\subsection{The Effects of Extra Data}

In the original train data, there are $764 \mathrm{k}$ person bounding boxes in 19 videos with $32.9 \mathrm{k}$ frames, and the testing set contains 13 videos with $15.1 \mathrm{k}$ frames. Considering the limited number of videos and duplicated image frames, the diversity of train data is not enough. And the train data and test data have many different scenes, thus extra data is crucial for training a superior detection model. Here we investigate the effects of different human detection dataset on HIE, including all the person images in $\mathrm{COCO}$ (COCO person, $64 \mathrm{k}$ images with $262 \mathrm{k}$ boxes) [12], CityPerson (2.9k image with $19 \mathrm{k}$ boxes) [27], CrowndHuman (15k images with 339k boxes) [18] and self-collected data ( $2 \mathrm{k}$ images with $30 \mathrm{k}$ boxes). We investigate the effects on different data based on Faster-RCNN with ResNet50 as the backbone. The experimental results are shown in Table 2. We can find that the CrowdHuman dataset achieves the largest improvement compared with other datasets, because the CrowdHuman is the most similar scenes with HIE, and both of the two datasets contain plenty of crowded scenes. COCO person contains two times of images than HIE train data, but merging the COCO person does not bring significant improvement and suffer more than three times train time, thus we only merge HIE with CrowdHuman and selfcollected data to take a trade-off between detection performance and train time.

\subsection{Detection in Crowded Scenes}

As there are lots of crowded scenes in HIE2020 dataset, the highlyoverlapped instances are hard to detect for the current detection framework. We apply a method aiming to predict instances in crowded scenes [2], named as "CrowdDet". The key idea of CrowdDet is to let each proposal predict a set of correlated instances rather than a single one as the previous detection method. The CrowdDet includes three main contributions for crowded-scenes detection: (1) an EMD loss to minimize the set distance between the two sets of proposals [20]; (3). Set NMS, it will skip normal NMS suppression when two bounding boxes come from the same proposal, which has been proved works in crowded detection; (2). A refine module that takes the combination of predictions and the proposal feature as input, then performs a second round of predicting. We conduct experiments to test the three parts on HIE2020 dataset, and the results are shown in Table 3. Based on the results in the Table, we can find that the three parts do improve the performance in crowded detection. Meanwhile, we apply KD regularization [26] in the class's logits of the detection model, which can consistently improve the detection results by $0.5 \%-1.4 \%$.

Finally, based on the above analysis, we train two detection models on HIE by combining extra data with the crowded detection framework: (1). ResNet152 + Cascade RCNN + extra data + emd loss + refine module + set NMS + KD regularization, whose AP is 83.21; (2). ResNeXt101 + Cascade RCNN + extra data + emd loss + refine module + set NMS + KD regularization, whose AP is 83.78 ; Then two models are fused with weights 1:1.
Table 1: Performance comparison (AP and $m M R$ ) among different detection backbone and methods on HIE dataset.

\begin{tabular}{l|c|c}
\hline Methods or Modules & AP (\%) & MMR (\%) \\
\hline Baseline (ResNet50 + Faster RCNN) & 61.68 & 74.01 \\
ResNet152 + Faster RCNN & 67.32 & 68.17 \\
ResNet152 + Faster RCNN + FPN & 69.77 & 64.83 \\
SENet154 + Faster RCNN + FPN & 65.77 & 68.46 \\
ResNeXt101 + Faster RCNN + FPN & 69.53 & 63.91 \\
ResNeXt101 + Cascade RCNN + FPN & 71.32 & 61.58 \\
ResNet152 + Cascade RCNN + FPN & 71.06 & 62.55 \\
\hline
\end{tabular}

Table 2: The effects of using extra data for human detection on HIE dataset.

\begin{tabular}{l|c|c}
\hline Validation set & AP (\%) & MMR (\%) \\
\hline HIE data & 61.68 & 74.01 \\
HIE + COCO person & 65.83 & 69.75 \\
HIE + CityPerson & 63.71 & 67.43 \\
HIE + CrowdHuman & $\mathbf{7 8 . 2 2}$ & $\mathbf{5 8 . 3 3}$ \\
HIE + self-collected data & $\mathbf{6 9 . 3 9}$ & $\mathbf{6 0 . 8 2}$ \\
HIE + CrowdHuman + COCO + CityPerson & 78.53 & 58.63 \\
HIE + CrowdHuman + self-collected data & $\mathbf{8 1 . 0 3}$ & $\mathbf{5 5 . 5 8}$ \\
HIE + all extra data & 81.36 & 55.17 \\
\hline
\end{tabular}

Table 3: Detection in Crowded Scenes on HIE dataset.

\begin{tabular}{l|c|c}
\hline Validation set & AP (\%) & MMR (\%) \\
\hline ResNet50 + Faster RCNN + extra data & 81.36 & 55.17 \\
+ emd loss & 81.73 & 53.20 \\
+ refine module & 81.96 & 50.85 \\
+ set NMS & 82.05 & 49.63 \\
\hline
\end{tabular}

\section{HUMAN TRACKING}

We adopted a classic two-stage multi-object tracking scheme composing of detection and box association. After the boxes are generated by the detection phase, DeepSORT [23] is applied to perform the association process and assign the id to the boxes. To improve on the baseline DeepSORT method, we used our own pretrained ReID model to extract deep appearance descriptors. For other parts, we retain a similar structure as described in [23]. Besides the improved use of a robust ReID feature extractor, we also made modifications in the following two perspectives.

\subsection{Optimal parameter search}

To obtain an optimal set of parameters for the tracking method to work well on the testing set, we performed a grid search on the 
Table 4: Grid search result on the training set of HIE dataset.

\begin{tabular}{c|c|c|c|c}
\hline \multicolumn{5}{c}{ Parameters } \\
\hline $\begin{array}{c}\text { max-cos-dis } \\
0.3\end{array}$ & nn-budget & max-age & nn-init & max-iou-dis \\
256 & 30 & 3 & 0.7 \\
\hline \multicolumn{5}{c}{ Performance on training set } \\
\hline MOTA & MOTP & FP & FN & ID Sw \\
87.7 & 0.131 & 24839 & 61677 & 10175 \\
\hline \multicolumn{5}{c}{ Performance on testing set } \\
\hline MOTA & MOTP & FP & FN & ID Sw \\
61.85 & 0.78 & 3710 & 21211 & 1657 \\
\hline
\end{tabular}

Table 5: Detection in Crowded Scenes on HIE dataset.

\begin{tabular}{l|c|c|c|c}
\hline Method & MOTA & FP & FN & ID Sw \\
\hline DeepSORT (Baseline) & 27.11 & 5894 & 42668 & 2220 \\
+ Own ReID \& Det & 53.46 & $\mathbf{5 6 2 2}$ & 24836 & 1963 \\
+ Horizontal flip & 53.50 & 5653 & 24798 & 1949 \\
+ Scale & $\mathbf{5 3 . 9 6}$ & 5922 & $\mathbf{2 4 5 0 1}$ & $\mathbf{1 6 5 7}$ \\
\hline
\end{tabular}

training set. The search space is designed for five parameters, respectively the maximum cosine distance (max-cos-dis), the number of object appearance history to keep (nn-budget), the max age of a track (max-age), the number of frames for a tentative track to be confirmed (nn-init), and the maximum IoU distance (max-iou-dis). The optimal parameter and results on both training and testing set are shown in Table 4. The performance in the table is also the performance of the final tracker that we used in pose tracking.

\subsection{Combination of ReID features}

We tested different strategies for combining ReID features to be used by the DeepSORT structure. (a) Linear combination of original features and features extracted from the horizontal flipped human; (b) Linear combination of ReID features extracted by models trained using different scales. The results on the testing set using a preliminary object detection result can be seen in Table 5 .

\section{POSE ESTIMATION}

\subsection{Framework}

Two state-of-the-art single-person pose estimation models are used in our method as the baseline, respectively HRNet [19] and SimpleNet [24]. FPN [9] is embedded into the structure of SimpleNet so that the performance on the small human bounding boxes can be improved. The results of HRNet and SimpleNet with FPN are then fused by averaging the heatmaps.
Table 6: Pose estimation result.

\begin{tabular}{c|c|c|c}
\hline & w_AP @ avg & w_AP @ 50 & w_AP @ 75 \\
\hline Ours & 56.34 & 69.60 & 53.02 \\
\hline
\end{tabular}

Table 7: Pose tracking result.

\begin{tabular}{c|c|c|c}
\hline & MOTA & MOTP & Total_AP \\
\hline Ours & 61.79 & 54.97 & 76.55 \\
\hline
\end{tabular}

\subsection{Optical Flow Smoothing}

The selected models consider the estimation of poses in different pictures as individual predictions. However, human actions are continuous in videos, which means the predictions in consecutive frames should be close to each other. Therefore, we advantage of the optical flow to improve the smoothness of our pose prediction.

We proposed to smooth the poses predicted in the current frame using the previous as well as next frame using optical flow. Given a human instance with joints coordinates set $J_{i}^{k-1}$ in the previous frame $I^{k-1}$, the estimated joints coordinates in the current frame $\hat{J}_{i}^{k-1 \rightarrow k}$ can be obtained using the optical field $F_{k-1 \rightarrow k}$ between $I^{k-1}$ and $I^{k}$ by propagating $J_{i}^{k-1}$ according to $F_{k-1 \rightarrow k}$. Specifically, for each joint location $(x, y)$ in $J_{i}^{k-1}$, the propagated joint location will be $(x+\delta x, y+\delta y$, where $\delta x, \delta y$ are the flow field values at joint location $(x, y)$. Similarly, we can estimate the current frame $\hat{J}_{i}^{k+1 \rightarrow k}$ from the next frame in the same way. Finally, we obtain the predicted $J_{i}^{k}$ as follows:

$$
J_{i}^{k}=\alpha \cdot \hat{J}_{i}^{k-1 \rightarrow k}+\alpha \cdot \hat{J}_{i}^{k+1 \rightarrow k}+(1-2 \alpha) \cdot J_{i}^{k}
$$

where the $J_{i}^{k}$ is the prediction of current frame from our pose estimation network and the $\alpha$ is used to weighted average the three terms.

The person identities used in optical flow smoothing is obtained using the tracking method described before. The final pose estimation score is presented in Table. 6 .

\subsection{Pose tracking}

The overall procedure for the pose tracking composes of several stages. (a) DeepSORT with our own ReID feature and private detection method is applied to generate ID for each person. (b) Human pose is individually estimated for each box in the result of human tracking. (c) Optical flow smoothing is performed as described, using the human id generated in (a) and human pose generated in (b). The final pose is obtained using Eq. 1. The identity of the pose is automatically transferred from the result of DeepSORT to the final poses with the corresponding bounding boxes. The final pose tracking result is presented in Table. 7. 


\section{CONCLUSION}

This paper presents a pipeline to solve the pose tracking problem in the video. The problem is decomposed into two separate problems that can be solved individually. First, for the human detection problem in crowded scenes, we investigate the effects of common detection frameworks on HIE2020 dataset, then we add extra data to overcome the overfitting problem and apply one proposal for multiple predictions to relieve the difficulty on detecting highlyoverlapping instances. After obtaining human positions, we apply DeepSORT to perform box association and assign ID for the boxes and made improvements on two perspectives, respectively in terms of parameter search and ReID features. Then we apply our effective single-person pose estimation model to generate accurate pose predictions and the predictions are smoothed by the proposed optical flow smoothing algorithm. The identity of the boxes are automatically transferred to the corresponding pose. Overall, the proposed pipeline performs well.

\section{REFERENCES}

[1] Zhaowei Cai and Nuno Vasconcelos. 2018. Cascade r-cnn: Delving into high quality object detection. In Proceedings of the IEEE conference on computer vision and pattern recognition. 6154-6162.

[2] Xuangeng Chu, Anlin Zheng, Xiangyu Zhang, and Jian Sun. 2020. Detection in Crowded Scenes: One Proposal, Multiple Predictions. In Proceedings of the IEEE/CVF Conference on Computer Vision and Pattern Recognition. 12214-12223.

[3] Piotr Dollar, Christian Wojek, Bernt Schiele, and Pietro Perona. 2011. Pedestrian detection: An evaluation of the state of the art. IEEE transactions on pattern analysis and machine intelligence 34, 4 (2011), 743-761.

[4] Ross Girshick. 2015. Fast r-cnn. In Proceedings of the IEEE international conference on computer vision. 1440-1448.

[5] Ross Girshick, Jeff Donahue, Trevor Darrell, and Jitendra Malik. 2014. Rich feature hierarchies for accurate object detection and semantic segmentation. In Proceedings of the IEEE conference on computer vision and pattern recognition. 580-587.

[6] Kaiming He, Georgia Gkioxari, Piotr Dollár, and Ross Girshick. 2017. Mask r-cnn In Proceedings of the IEEE international conference on computer vision. 2961-2969.

[7] Kaiming He, Xiangyu Zhang, Shaoqing Ren, and Jian Sun. 2016. Deep residual learning for image recognition. In Proceedings of the IEEE conference on computer vision and pattern recognition. $770-778$.

[8] Jie Hu, Li Shen, and Gang Sun. 2018. Squeeze-and-excitation networks. In Proceedings of the IEEE conference on computer vision and pattern recognition. 7132-7141.

[9] Tsung-Yi Lin, Piotr Dollár, Ross Girshick, Kaiming He, Bharath Hariharan, and Serge Belongie. 2017. Feature pyramid networks for object detection. In Proceedings of the IEEE conference on computer vision and pattern recognition. 2117-2125.

[10] Tsung-Yi Lin, Piotr Dollár, Ross Girshick, Kaiming He, Bharath Hariharan, and Serge Belongie. 2017. Feature pyramid networks for object detection. In Proceedings of the IEEE conference on computer vision and pattern recognition. 2117-2125.

[11] Tsung-Yi Lin, Priya Goyal, Ross Girshick, Kaiming He, and Piotr Dollár. 2017. Focal loss for dense object detection. In Proceedings of the IEEE international conference on computer vision. 2980-2988.

[12] Tsung-Yi Lin, Michael Maire, Serge Belongie, James Hays, Pietro Perona, Deva Ramanan, Piotr Dollár, and C Lawrence Zitnick. 2014. Microsoft coco: Common objects in context. In European conference on computer vision. Springer, 740-755.

[13] Weiyao Lin, Huabin Liu, Shizhan Liu, Yuxi Li, Guo-Jun Qi, Rui Qian, Tao Wang Nicu Sebe, Ning Xu, Hongkai Xiong, et al. 2020. Human in Events: A Large-Scale Benchmark for Human-centric Video Analysis in Complex Events. arXiv preprint arXiv:2005.04490 (2020).

[14] Wei Liu, Dragomir Anguelov, Dumitru Erhan, Christian Szegedy, Scott Reed, Cheng-Yang Fu, and Alexander C Berg. 2016. Ssd: Single shot multibox detector. In European conference on computer vision. Springer, 21-37.

[15] Joseph Redmon, Santosh Divvala, Ross Girshick, and Ali Farhadi. 2016. You only look once: Unified, real-time object detection. In Proceedings of the IEEE conference on computer vision and pattern recognition. 779-788.

[16] Joseph Redmon and Ali Farhadi. 2016. YOLO9000: Better, Faster, Stronger. arXiv preprint arXiv:1612.08242 (2016).

[17] Shaoqing Ren, Kaiming He, Ross Girshick, and Jian Sun. 2015. Faster r-cnn Towards real-time object detection with region proposal networks. In Advances in neural information processing systems. 91-99.

[18] Shuai Shao, Zijian Zhao, Boxun Li, Tete Xiao, Gang Yu, Xiangyu Zhang, and Jian Sun. 2018. Crowdhuman: A benchmark for detecting human in a crowd. arXiv preprint arXiv:1805.00123 (2018)

[19] Ke Sun, Bin Xiao, Dong Liu, and Jingdong Wang. 2019. Deep high-resolution representation learning for human pose estimation. In Proceedings of the IEEE conference on computer vision and pattern recognition. 5693-5703.

[20] Siyu Tang, Mykhaylo Andriluka, and Bernt Schiele. 2014. Detection and tracking of occluded people. International fournal of Computer Vision 110, 1 (2014), 58-69.

[21] Tao Wang, Li Yuan, Xiaopeng Zhang, and Jiashi Feng. 2019. Distilling object detectors with fine-grained feature imitation. In Proceedings of the IEEE Conference on Computer Vision and Pattern Recognition. 4933-4942.

[22] Tao Wang, Xiaopeng Zhang, Li Yuan, and Jiashi Feng. 2019. Few-shot adaptive faster r-cnn. In Proceedings of the IEEE Conference on Computer Vision and Pattern Recognition. 7173-7182.

[23] Nicolai Wojke, Alex Bewley, and Dietrich Paulus. 2017. Simple online and realtime tracking with a deep association metric. In 2017 IEEE international conference on image processing (ICIP). IEEE, 3645-3649.

[24] Bin Xiao, Haiping Wu, and Yichen Wei. 2018. Simple baselines for human pose estimation and tracking. In Proceedings of the European conference on computer vision (ECCV). 466-481.

[25] Saining Xie, Ross Girshick, Piotr Dollár, Zhuowen Tu, and Kaiming He. 2017. Aggregated residual transformations for deep neural networks. In Proceedings of the IEEE conference on computer vision and pattern recognition. 1492-1500.

[26] Li Yuan, Francis EH Tay, Guilin Li, Tao Wang, and Jiashi Feng. 2019. Revisit knowledge distillation: a teacher-free framework. arXiv preprint arXiv:1909.11723 (2019).

[27] Shanshan Zhang, Rodrigo Benenson, and Bernt Schiele. 2017. Citypersons: A diverse dataset for pedestrian detection. In Proceedings of the IEEE Conference on Computer Vision and Pattern Recognition. 3213-3221.

[28] Li Zhou, Jian Zhao, Jianshu Li, Li Yuan, and Jiashi Feng. 2018. Object Relation Detection Based on One-shot Learning. arXiv preprint arXiv:1807.05857 (2018). 